\title{
Comparison of Shack-Hartmann sensor and Point Diffraction Interferometer for Wavefront Aberration analysis.
}

\author{
A. Marzoa, S. Bosch and S. Vallmitjana \\ Applied Physics Department \\ Faculty of Physics, University of Barcelona \\ Barcelona, Spain \\ tonymarzoa4@gmail.com
}

\begin{abstract}
Two methods for assessing the image quality of optical systems based on the analysis of wavefront aberration (WA) are presented together. The methods and the experimental setups are broadly described. A comparative study between those two techniques on the analysis of a meniscus lens has been carried out and an equivalence between both techniques has been tested and demonstrated, by comparing the RMS associated to their measured Zernike coefficients.
\end{abstract}

Keywords-wavefront; aberration; optical testing; Zernike polynomials; Shack-Hartmann; Point diffraction interferometer

\section{INTRODUCTION}

Measuring and analyzing the quality of optical systems for their use in different fields and applications is an interesting area of continuous development. Although a large number of techniques are available for optical testing, interferometry and wavefront sensing techniques have been commonly used in recent years.

Since it was proposed for use in astronomy ShackHartmann wavefront sensor (SH) [1] has been extensively used in many areas, such as adaptive optics and ophthalmology [2]. On the other hand, in the last few years, the point-diffraction interferometer (PDI) [3] has a growing use in areas like ophthalmology because of its simplicity and high dynamical range [4].

In this paper a comparative study between those two techniques on the analysis of a meniscus lens has been carried out. Equivalence between both techniques has been tested and demonstrated, obtaining a difference between the associated RMS corresponding to both techniques of $0.08 \mu \mathrm{m}$. A similar equivalence has been reported before by Bueno et al. [5] on the study of phase plates but not in the case of real focusing systems. The study allows us to compare and check out the equivalences and the similarity of results and points out the advantages and difficulties of each method. At the same time the work has given us good training ground for developing and deepening our knowledge of both techniques.

\section{THEORY}

It is well known that through the geometrical parameters and refractive indices of the components that compose an optical system it is possible to determine the position and the size of the image of an object seen through this optical system. Nowadays there are several commercial software platforms, operating by modeling ray tracing or by optical propagation calculations, that can provide different features.

As a general concept, geometrical aberrations can be defined as the deviation that a wavefront undergoes at the exit pupil (EP) of the system with respect the ideal. When the conditions are fitted in the case of an image forming system, the ideal wavefront is spherical.

An interesting mathematical tool to describe optical aberrations is the set of Zernike polynomials introduced by Fritz Zernike in 1934. Zernike polynomials, expressed in polar coordinates are orthogonal in the unit circle. Using this set of polynomials the WA could be written as [6]:

$$
W(\rho, \theta)=\sum_{n=0}^{\infty} \sum_{m=0}^{n} C_{n m} Z_{n}^{m}(\rho, \theta)
$$

A common metric of wavefront flatness for the WA is the RMS wavefront error which can be computed in terms of Zernike coefficients using [2]

$$
R M S=\left(\sum_{j=3}^{N} C_{j}^{2}\right)^{1 / 2}
$$

\section{A. Shack-Hartmann wavefront sensor}

A Shack-Hartmann (SH) wavefront sensor basically consists of a microlens array and a CCD camera. The wavefront is divided by the microlens in such a way that each light division is brought to a particular location in the focal plane of the array. If there is aberration in the wavefront, these spots shift from the regularly spaced ideal spot pattern produced by a plane

978-1-5090-2163-5/16/\$31.00 2016 IEEE. 
wavefront. These displacements $(\Delta \mathrm{x}$ and $\Delta \mathrm{y})$ provide information on the local slope or tilt of the wavefront over each microlens. Any phase aberration can be approximated by a set of discrete tilts. By sampling the array of microlens, all of these tilts can be measured and the whole wavefront approximated. Commercial Shack-Hartmann sensors can reconstruct the WA by using the software provided by the manufacturer.

\section{B. Point-diffraction interferometer}

The Point-diffraction interferometer (PDI) is a simple common-path interferometer used to directly measure optical path differences. A PDI basically consists of a semitransparent plate with a clear pinhole.

When a light beam reaches the plate, a spherical reference wave is produced by diffraction at the clear pinhole while the rest of the beam passes through the plate without any change in phase. If the size of the pinhole and the transmittance of the plate are chosen in such a way both beams have similar amplitude, well contrasted fringes will be observed in any plane placed after the plate. The interferogram provides information for calculation the WA.

\section{EXPERIMENTAL SETUP}

Combining the two techniques explained before, two different experimental set-ups were designed. On the one hand there is a set-up for measuring the WA at a certain plane after the focal plane of the system under study. On the other hand there is a set-up designed in order to measure the WA at the EP of the system.

In both cases, a meniscus ophtalmic lens with a focal length of $200 \mathrm{~mm}(+5 \mathrm{D})$ was tested by using the SH and the PDI techniques. The SH measurements were computed by the software provided by the device's manufacturer. PDI interferograms were analyzed using Atmosfringe's software [7].

\section{A. Measuring the $W A$ at a certain plane $z$}

In order to compare both techniques, it was important that the observation plane of the PDI and the pupil size of SH should have the same diameter. This means that Zernike coefficients computed by the two methods had the same scale and then we were measuring the same WA.

So, for an observation plane defined by the best configuration for the PDI, some images of the interferograms were captured. Then, the PDI configuration was replaced by the $\mathrm{SH}$ wavefront sensor and a measurement with this instrument was taken. A sketch of the experimental set-up used in this section is shown in Fig. 1.

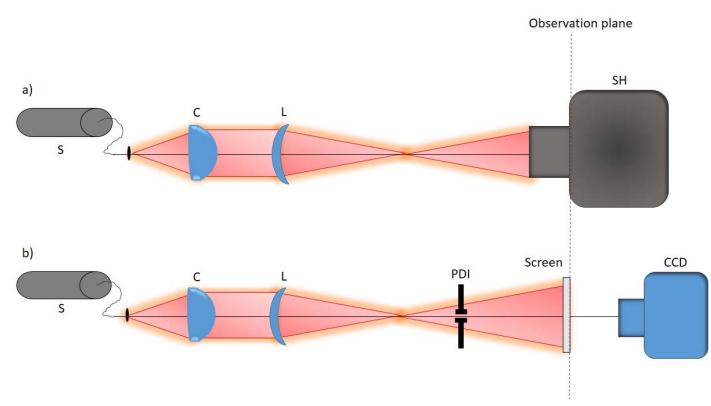

Fig. 1. Experimental setup used in this section for measuring the WA at a certain plane $z$ from the system under study. a): Using Shack-Hartmann sensor. He-Ne laser and monomode and optical Fiber (S). Collimator system (C), lens under test (L). SH wavefront sensor (SH) in plane $z$. b) Using Point Diffraction Interferometer. The same initial components and the PDI plate, screen to project the interferogram and a CCD camera in plane $z$. The observation plane of the screen has the same size as the entrance pupil of the $\mathrm{SH}$ wavefront sensor.

\section{B. Measuring the $W A$ at the EP of the system}

In order to measure the WA at the EP of the system under test a new setup was created by using an auxiliary system which optically conjugated the EP into the sensor or in the screen. A schematization of the setup is presented in Fig. 2.

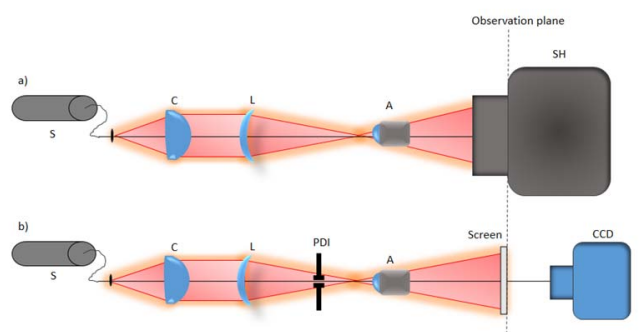

Fig. 2. E experimental setup used in this section for measuring the WA at the EP of the meniscus lens under test. The setup is the same of presented in Fig. 1., but there is an auxiliary system (A) which optically conjugated the EP of the (L) in the sensor in case a) or on the screen in case b).

\section{RESULTS}

Using the Zernike polynomial set obtained by each measure with the experimental procedures explained before, the WA can be computed. Results of this simulation are shown in Fig. 3. A visual inspection shows that both techniques provide similar results. Although tilts were always excluded, a minimal remaining tilt was present.

Using (2) and excluding defocus the RMS wavefront error can be computed. When coefficients from the $4^{\text {th }}$ to $24^{\text {th }}$ order of the expansion are taken, the total RMS between both techniques is reduced to $0.08 \mu \mathrm{m}$.

In order to analyze the behavior of the WA obtained in a set of planes close to the focal zone of the system under study, the 
Point-Spread Function (PSF) was computed by using the Zernike coefficients obtained by the $\mathrm{SH}$ and PDI measurements, and propagated forward and backwards. Results of this simulation and the experimentally captured corresponding PSFs are presented in Fig. 4, it is clear that results provided by both techniques agree and present the same behavior as the real WA.

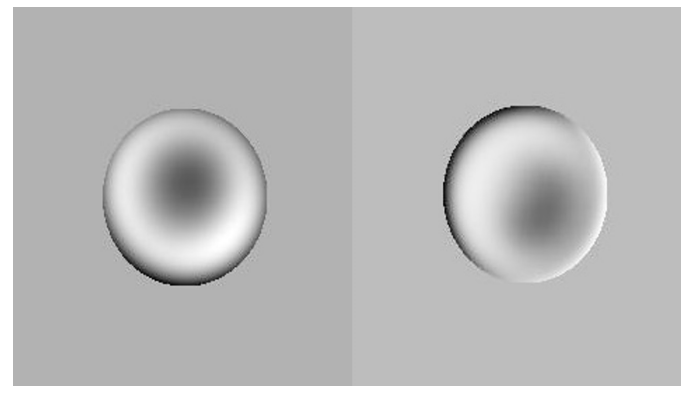

Fig. 3. Wavefront reconstructed using SH (left) and PDI (right) data.

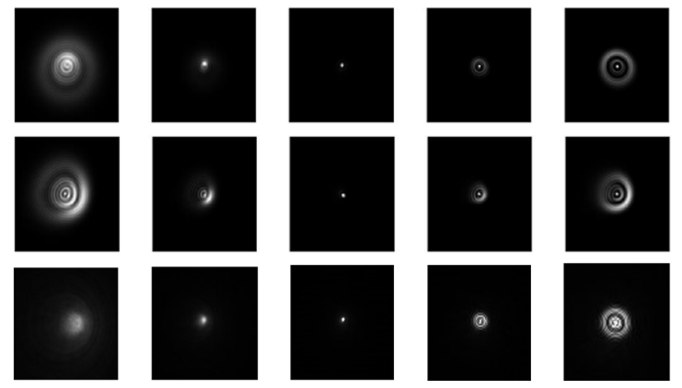

Fig. 4. Propagation of WA's PSF in the vicinities of the focal plane for SH (first row) and PDI (second row) measurements. Third row shows the experimental PSF captured with a CCD camera at the same set of planes of the simulation.

\section{DISCUSSION}

Despite the different nature of both techniques used in this work it is possible to obtain complementary information of the WA of an optical system using a set-up combining these two methods.

On one hand, the use of PDI is a very simple technique. The principal problem in the image processing of the interferogram provided by the PDI resides in the fact that depending on the location of the plate with the pin-hole it could affect the calculation of coefficients and also noise in comparison with the other interferometric measurement.

On the other hand, the SH is a robust and well-known device used in the study of optical aberrations. It presents an advantage over the PDI as it provides a more accurate reconstruction of the WA, a real time alignment capability and no need to use coherent light. But it presents the drawback that it is not possible to detect variations smaller than the size of the microlenses, and when the relative aperture is large, precision diminishes.

Moreover, advantages and disadvantages presented by each technique could be compensated using the other one and then a more accurate and complete reconstruction of WA will be obtained.

\section{CONCLUSIONS}

We have demonstrated the equivalence between the WA of a focusing system measurements of SH and PDI. These results could enable us to study the WA in a deeper way in many kinds of optical systems such as ophthalmic lenses, antique optical instruments like telescopes and microscopes, and biological samples such as crystalline lenses of animal eyes.

Moreover, the experimental procedure and the computational tools presented and used in this work would be an interesting support in order to explore and analyze the propagation of light and the propagation and analysis of aberrated images.

\section{Acknowledgment}

This work was supported by the Spanish Ministerio de Economía y Competitividad, project FIS2012-38244.

\section{References}

[1] B. C. Platt, R. Shack."History and Principles of Shack-Hartmann Wavefront Sensing”, Journal of Refractive Surgery, vol. 17, 2001.

[2] P.M. Prieto et al. "Analysis of the performance of the Hartmann-Shack sensor in the human eye "J J Opt Soc Am (A), 17, 8 1388-1398, 2000.

[3] R. N. Smartt and W. H. Steel, "Theory and application of point diffraction interferometers," Jpn. J. Appl. Phys. 14, 351-356 (1975).

[4] E. Acosta, S. Chamadoira, R. Blendowske, “Modified point diffraction interferometer for inspection and evaluation of ophthalmic components".J Opt Soc Am (A), 23,:632-7, 2006.

[5] J. M. Bueno et al, "Wavefront measurements of phase plates combining a point-diffraction interferometer and a Hartmann-Shack sensor," Applied Optics, vol. 49, 2010.

[6] V. Lakshminarayanan et al, "Zernike polynomials: a guide," Journal of Modern Optics, vol 58, 2011.

[7] Atmosfringe software written by Massimo Riccardi. http://www.atmossoftware.it/AtmosFringe.html 